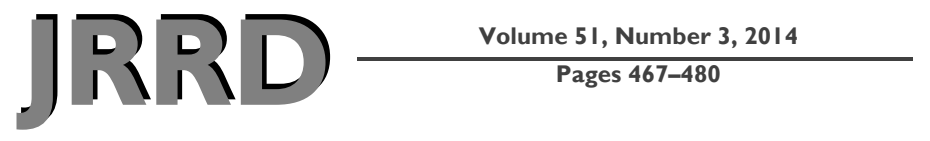

\title{
Effect of dynamic keyboard and word-prediction systems on text input speed in persons with functional tetraplegia
}

\author{
Samuel Pouplin, OT, MSc; ${ }^{1-3 *}$ Johanna Robertson, PT, PhD; ${ }^{2-3}$ Jean-Yves Antoine, PhD; ${ }^{4}$ Antoine Blanchet, \\ DI; ${ }^{5}$ Jean Loup Kahloun, Development Engineer; ${ }^{5}$ Philippe Volle, Director; ${ }^{6}$ Justine Bouteille, OT; $^{1-3}$ Frédéric \\ Lofaso, MD, PhD; ${ }^{2-3,7}$ Djamel Bensmail, MD, PhD ${ }^{1-3}$ \\ ${ }^{1}$ New Technologies Plate-Forme and ${ }^{2}$ Physical Medicine and Rehabilitation Department, AP-HP, Raymond Poincaré \\ Teaching Hospital, Garches, France; ${ }^{3}$ EA 4497 and Technological Innovations Centre (Inserm U 805), Versailles St- \\ Quentin-en-Yvelines University, Garches, France; ${ }^{4}$ Computer Lab, François Rabelais University, Tours \& Lab-STICC, \\ CNRS, Lorient, France; ${ }^{5}$ In Vienetis Inc, Paris, France; ${ }^{6}$ IN'TECH INFO, ESIEA, Ivry sur Seine, France; \\ ${ }^{7}$ Physiology-Functional Testing Ward, AP-HP, Raymond Poincaré Teaching Hospital, Garches, France
}

\begin{abstract}
Information technology plays a very important role in society. People with disabilities are often limited by slow text input speed despite the use of assistive devices. This study aimed to evaluate the effect of a dynamic on-screen keyboard (Custom Virtual Keyboard) and a word-prediction system (Sibylle) on text input speed in participants with functional tetraplegia. Ten participants tested four modes at home (static onscreen keyboard with and without word prediction and dynamic on-screen keyboard with and without word prediction) for 1 mo before choosing one mode and then using it for another month. Initial mean text input speed was around 23 words per minute with the static keyboard and 12 words per minute with the dynamic keyboard. The results showed that the dynamic keyboard reduced text input speed by $37 \%$ compared with the standard keyboard and that the addition of word prediction had no effect on text input speed. We suggest that current forms of dynamic keyboards and word prediction may not be suitable for increasing text input speed, particularly for subjects who use pointing devices. Future studies should evaluate the optimal ergonomic design of dynamic keyboards and the number and position of words that should be predicted.
\end{abstract}

Key words: assistive technology, computer, dynamic keyboard, learning, quadriplegia, satisfaction, self-help devices, text input speed, virtual keyboard, word-prediction system.

\section{INTRODUCTION}

Computers now play an important role in the lives of most individuals. They are used for recreational purposes (e.g., multimedia and games), work, and communication (e.g., Internet, email, instant messages) [1]. Access to computers is crucial for people with disabilities and may improve their quality of life [2]. The use of computers can facilitate mainstreaming at school, for example, and the Internet may provide a valuable means of communication [3-4]. However, the use of computers requires a certain degree of motor ability. People with motor disabilities frequently experience difficulties using a standard keyboard and standard pointing input systems such as a mouse. Many solutions exist to facilitate computer

\footnotetext{
Abbreviations: CVK = Custom Virtual Keyboard, SD = standard deviation, VAS $=$ visual analog scale, $\mathbf{w p m}=$ words per minute.

*Address all correspondence to Samuel Pouplin, OT, MSc; Ergothérapeute MSc, Plate-Forme Nouvelles Technologies, Hôpital R. Poincaré, 104 boulevard R. Poincaré, 92380 Garches, France +33147107061; fax: +33147107063.

Email: samuel.pouplin@rpc.aphp.fr

http://dx.doi.org/10.1682/JRRD.2012.05.0094
} 
access, depending on the person's specific impairments and the purpose for which the computer is used [5-8]. The most common solution relies on the use of a virtual keyboard that is directly displayed on the computer screen. The selection of the desired key on the virtual keyboard can be handled by a large variety of input devices, from a microgravity mouse to single switch devices supplemented by a process of dynamic scanning of the keyboard.

Although such assistive devices render computers accessible to people with disabilities, the actual inputting of text can be very slow. In a review of the literature, Le Pévédic found that nondisabled subjects typing with one finger had a text input speed of 11 words per minute (wpm), which increased to 15 wpm with several fingers; secretaries (using 10 fingers) had a speed of 25 wpm [9]. For subjects with disabilities using an assistive device, text input speed was only 5 wpm. Over the past few years, attempts have been made to develop systems to improve text input speed.

One method is to optimize the layout of standard keyboards [10] and static on-screen keyboards, for example based on bigrams of words, in order to reduce the number of movements necessary when using pointing devices or the number of selections necessary when using switches. Simulation studies have shown that optimizing the layout of the keyboard can increase text input speed by 36-55 percent compared with a QWERTY keyboard [11-12]. A study in five nondisabled subjects using pointing devices to input text on an optimized OPTI keyboard showed that the optimization of the position of the letters increased text input speed by 35 percent compared with a QWERTY keyboard [13], likely because the distance between letters was reduced, although some learning time was required.

Ambiguous and dynamic keyboards have also been developed to increase text input speed. Ambiguous keyboards combine several letters on the same key, like on mobile telephones. For people using pointing devices, this reduces the displacement between two keys and therefore reduces the motor cost. Equally, for persons who scan, the time necessary to select the key is reduced [14].

Lesher et al. showed the importance of the position of the letters on ambiguous keyboards independently from the prediction algorithm using a simulation [15]. For subjects using pointing devices, Vigouroux et al. evaluated nondisabled subjects and subjects with disabilities and found that for both groups, text input speed was faster using an AZERTY keyboard than a T9 ambiguous keyboard [16]. For subjects using scanning systems, a simulation by Harbusch and Kühn found a reduction of 53-60 percent in the number of selection steps when using an ambiguous keyboard compared with an optimized keyboard, whatever the type of scanning system used (linear scanning or row-column scanning methods) [17]. However in the case of row-column scanning, the improvement was smaller (10\%). Although ambiguous keyboards reduce the number of selection steps, the motor cost is greater because of the greater number of selection clicks necessary [14].

In dynamic keyboards, the layout of the keyboard is altered at each key press so that the characters most likely to follow are positioned around the one that has just been typed. For people who use scanning devices, dynamic keyboards have been shown to reduce the number of key selections necessary or the latency between two selections; for people using pointing devices, dynamic keyboards have been shown to reduce the displacement of the cursor. Text input speed has been shown to be the same using a dynamic keyboard as a QWERTY keyboard in nondisabled subjects [18] and to increase by 20 percent in subjects with disabilities using pointing devices with a dynamic Spreadkey keyboard compared with a QWERTY keyboard [19]. However, in a study which included persons who scan, Schadle et al. found an increase of 32 percent for text input time and 50 percent for the number of validations using a dynamic (linear) scanning system in comparison with a keyboard with a frequency-based organization line/column in a simulation study [20]. However, very few studies have evaluated the effect of such keyboards on text input speed in participants with motor disability over a long duration.

Another method to increase text input speed is to display words that are predicted from the letters previously typed. Word prediction reduces the number of necessary key strokes by avoiding having to type the whole word. Indeed, Higginbotham found keystroke savings of 3148 percent in a simulation study using word prediction in five different types of communication software for people with disabilities [21]. The effect on text input speed is, however, uncertain and results in the literature are inconclusive. In the case of pointing devices, Anson found that when nondisabled subjects use a physical keyboard, there is a reduction in text input speed if they use word prediction (mean $49.55 \mathrm{wpm}$ reduced to mean 13.95 wpm) [22]. However, when nondisabled subjects 
use a virtual keyboard, text input speed increases from $8.7 \mathrm{wpm}$ without word prediction to 9.4-11.2 wpm with word prediction. Similarly, Koester and Levine found that word prediction did not improve text input speed in nondisabled subjects using a physical keyboard and reduced text input speed by 41 percent in subjects with spinal cord injury [23]. With regard to scanning systems, Koester and Levine found that word prediction reduced text input speed in nondisabled subjects (by 8.2\%) [2425]. Heckathorne et al. showed that the greater the visual search time (related to the number of predicted words presented), the greater the reduction in text input speed in three subjects with disabilities [26].

The aim of this study was to carry out a preliminary evaluation of a dynamic on-screen keyboard and a wordprediction system (Custom Virtual Keyboard [CVK]) on text input speed in participants with functional tetraplegia using the systems at home for 2 mo. The CVK was developed by our team and is available free of charge (Figure 1).

We hypothesized that both word prediction and the dynamic keyboard would increase text input speed and, thus, the combination of both systems would further increase text input speed.

\section{METHODS}

\section{Participants}

Participants with functional tetraplegia seen at the Physical Medicine and Rehabilitation department of the Raymond Poincaré Teaching Hospital (Garches, France) between 2005 and 2010 were contacted by telephone to determine whether they fulfilled the inclusion criteria and wished to participate. Participants were included if they were over $18 \mathrm{yr}$ old, had functional tetraplegia (e.g., due to locked-in syndrome, myopathy, or cervical spinal cord injury), regularly used an on-screen static AZERTY keyboard based on a personal computer with Windows (Microsoft Corp; Redmond, Washington) (the only operating system that can accommodate the CVK at present), and were not regular users of dynamic keyboards or word prediction. Participants had home access to the Internet and lived in or near Paris, France. Participants were excluded if they had cognitive, linguistic, or visual impairments preventing the use of a computer.

\section{Materials}

This study was carried out on the CVK, which was developed by our team and is available as open-source software (Figure 1).

Text input using the CVK can be achieved using pointing devices or, for participants with too little motor capacity to use a pointing device, via automatic scanning. When a pointing device is used, the user positions the cursor using a pointing device over the desired virtual key and then validates the choice. This type of mode fits, for instance, the needs of people with functional tetraplegia who use a head pointing device. For people who can only control their physical environment by means of a single switch, an automatic process enables the cursor to successively scan all the relevant positions of the screen. When the intended key is reached by the cursor, the user validates that key using a switch. This form of text input is, however, very slow. Two types of automatic scanning modes were used in this study: row-column and linear. The row-column mode significantly reduces the number of cursor shifts needed to reach the intended key but requires two keystrokes (line and column) to select each item, thus increasing the physical effort of the user. Linear scanning requires only a single keystroke because all the keys are systematically scanned successively. When used with a static AZERTY keyboard, text input speed is

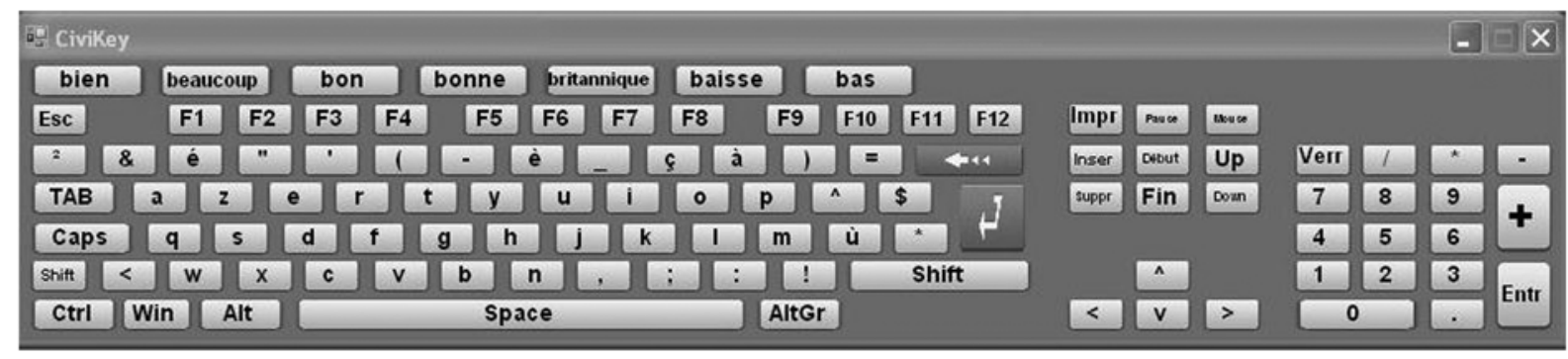

Figure 1.

Custom Virtual Keyboard on-screen keyboard. 
therefore dramatically reduced if the intended key is situated at the end of the keyboard.

Two types of keyboards exist within the CVK: a standard on-screen static AZERTY keyboard and a dynamic on-screen keyboard. The dynamic mode is based on the Sibylle augmentative and alternative communication system [27] and consists of an automatic rearrangement of the characters on the keyboard after each selection such that the characters that are most likely to be typed next are displayed next to the character that has just been typed, taking into account the previously selected letters. This rearrangement is achieved by the stochastic letter prediction module of Sibylle, which was trained on a large corpus of around 100 million words. Figure 2 illustrates this dynamic modification of the keyboard display (English version of Sibylle) when the user tries to write the word three. At first, the letters are set in the following order: $t$, $a, i, s, o$, etc. The letter $t$ is the most frequent letter that begins a word in the trained corpus. When, the user selects the letter $t$, the keyboard is automatically rearranged in the following new order: $h, o, r, e$, a, etc. Here, the letter $h$ is proposed first because it is the most likely to occur after the letter $t$. In other words, the conditional probability $\mathrm{P}\left(\mathrm{l}_{\mathrm{i}} \mid \mathrm{l}_{\mathrm{i}-1}=t\right)$ is maximum, with $\mathrm{l}_{\mathrm{i}}=h$. The letter prediction module of the CVK is based on a 5-g language model $\mathrm{P}\left(\mathrm{l}_{\mathrm{i}} \mid \mathrm{l}_{\mathrm{i}-1}, \mathrm{l}_{\mathrm{i}-2}, \mathrm{l}_{\mathrm{i}-3}, \mathrm{l}_{\mathrm{i}-4}\right)$, which means that the system considers the last four selected letters for the reorganization of the keyboard layout.

Theoretically, this dynamic keyboard should speed up the access time to the intended key and thus increase text input speed. As noted in the introduction, text input speed can also be increased by means of word prediction in order to reduce the number of keystrokes required.

\begin{tabular}{|c|c|c|c|c|c|c|c|}
\hline$-\infty$ & Word & \pm & a & i & $s$ & (x) & before $t$ \\
\hline 0 & $w$ & b & h & $c$ & $p$ & $f$ & \multirow{3}{*}{ after $t$} \\
\hline$\infty$ & Word & h. & 0 & $r$ & e & (x) & \\
\hline a & i & $w$ & u & $y$ & $v$ & - & \\
\hline $1 \infty$ & Word & e & $a$ & i & 0 & $1 \times$ & \multirow{2}{*}{ after $t h$} \\
\hline$r$ & $\mathbf{u}$ & $w$ & - & $y$ & $\dagger$ & $s$ & \\
\hline
\end{tabular}

Figure 2.

Rearrangement of dynamic keyboard (central part of keyboard shown). (English version of Sibylle.)
The CVK (Figure 1) includes a word-prediction module that is based on SibySem, a context-sensitive prediction module that has been shown to reach state-of-the-art performance in French, English, and German [28]. This module is not based on a simple dictionary like standard commercial systems. It is based on a language model that considers the last two words already typed as well as the semantic context of the message. New words are learned dynamically by the system as input continues. Moreover, the system gradually learns the language style of the user. This prediction system is innovative in that word prediction is based on the lexical meaning of the sentence. This characteristic allows the prediction to adjust dynamically to the current topic of interest. Experiments with participants have shown that the word-prediction system can achieve about 60 percent keystroke savings [28] when five predicted words are displayed at a time.

The prediction list is displayed horizontally at the top of the virtual keyboard in Figure 1 (bien, beaucoup, bon, etc.) and vertically on the left of the keyboard in Figure 3.

\section{Custom Virtual Keyboard Modes}

In this study, four different modes of the CVK software were compared:

- Static on-screen keyboard.

- Static on-screen keyboard with word prediction.

- Dynamic on-screen keyboard.

- Dynamic on-screen keyboard with word prediction.

The static mode consisted of a virtual keyboard with the standard AZERTY layout. The static+word-prediction mode consisted of this virtual AZERTY keyboard coupled with the Sibylle word-prediction system. The wordprediction display was located at the top of the on-screen keyboard and presented seven words (Figure 1). The scanning system integrated within the static keyboard was row-column. The dynamic mode consisted of a virtual keyboard whose layout changed after each character input to display the characters most likely to be selected next. In the dynamic+word-prediction mode, Sibylle was used in addition to the dynamic keyboard. The word-prediction display was located to the left of the dynamic keyboard and presented five words (Figure 3). The scanning system integrated within the dynamic keyboard was linear.

\section{Study Design}

This was a pilot study for which ethical approval was not necessary according to French law, because it was an evaluation of usual practice. 


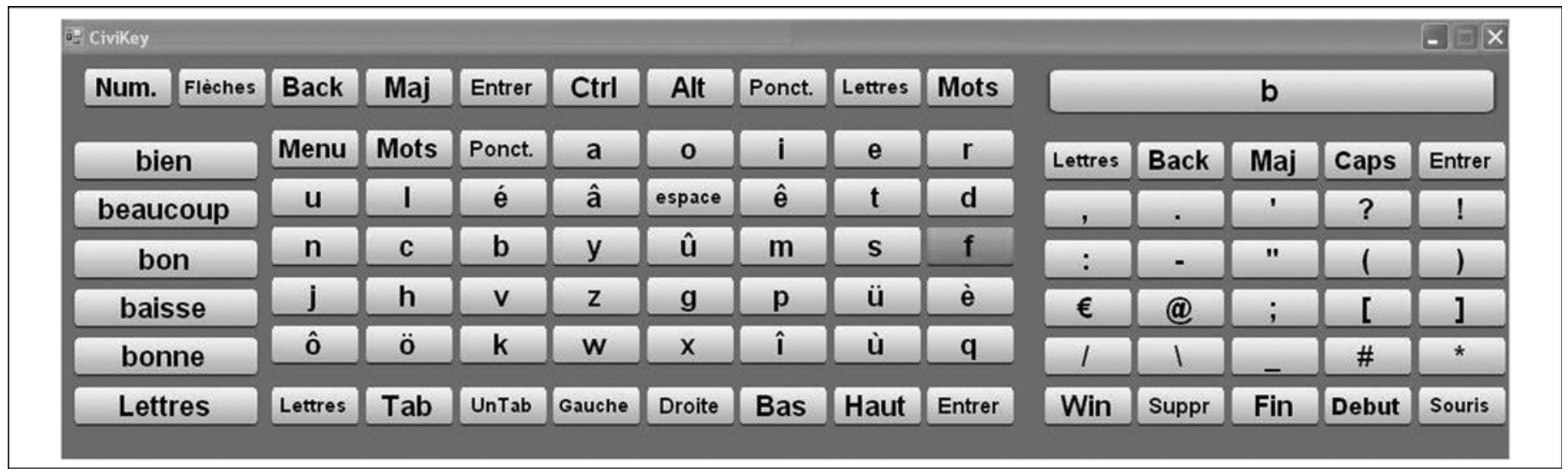

Figure 3.

Custom Virtual Keyboard dynamic on-screen keyboard with word-prediction list on left (French version).

The study was carried out over 2 mo. The CVK was downloaded on each participant's computer. The participants used their usual interfaces (e.g., trackball, switch, mouse, joystick, or head-controlled device). Specific software was coupled with the CVK to record quantitative data, such as software use in hours per day and number of characters typed.

An experienced occupational therapist (S. Pouplin) spent $1 \mathrm{~h}$ with each participant to explain the function of the four study modes. The rationale behind word prediction and dynamic keyboards was explained, but subjects were not given specific guidelines or strategies regarding their use. During the first month, the participants tested the four CVK modes.

The modes opened randomly with each CVK session. However, the participants could close the currently opened mode, thus obtaining access to another mode, and could therefore completely avoid the use of one or more modes should they wish to. This choice was made because we felt it was unfair to limit the participants to the use of a mode that they found restrictive. We were conscious that times of use during the study were therefore likely not to be equal.

At the end of the first month, the occupational therapist returned to the participant's home to carry out the assessment. The participant then chose the mode he or she preferred and used it for the next month.

\section{Assessment}

Three evaluation sessions were carried out: at baseline (D0), at the end of the first month (D30), and at the end of the second month (D60) (Figure 4).
All the modes of the CVK were evaluated in a random order at D0 and D30. For the assessment at D60, only the chosen CVK mode was evaluated. During the evaluation sessions, input speed during a copying task was evaluated with a 400-word text that the participant was asked to type in less than $10 \mathrm{~min}$. Participants were instructed to use the word prediction and the dynamic keyboard as desired; i.e., no instructions regarding strategies of use were given. Four texts of similar complexity were used, drawn from national newspapers with an average word length of 5.3 characters \pm 0.3 (standard deviation [SD]). The texts were randomly allocated in order to ensure that the same text was not associated with the same CVK mode.

\section{Outcome Measures}

During the three evaluations, the text input speed (calculated as time taken to copy the text/number of characters typed, including punctuation marks and spaces but not including backspace, selection errors, and correction times) was calculated for each mode at each session. At the D30 and D60 evaluations, satisfaction was evaluated using a $0-10$ visual analog scale (VAS). On D30, the participants were asked to classify the four modes in order of their preference.

In addition to these evaluation sessions, the CVK automatically recorded time of use of the device by the participants in their home environments outside of the evaluation sessions. The recording began as soon as the cursor of the mouse moved in the zone of the on-screen keyboard and stopped when the cursor moved out of the keyboard area or was static over the on-screen keyboard 


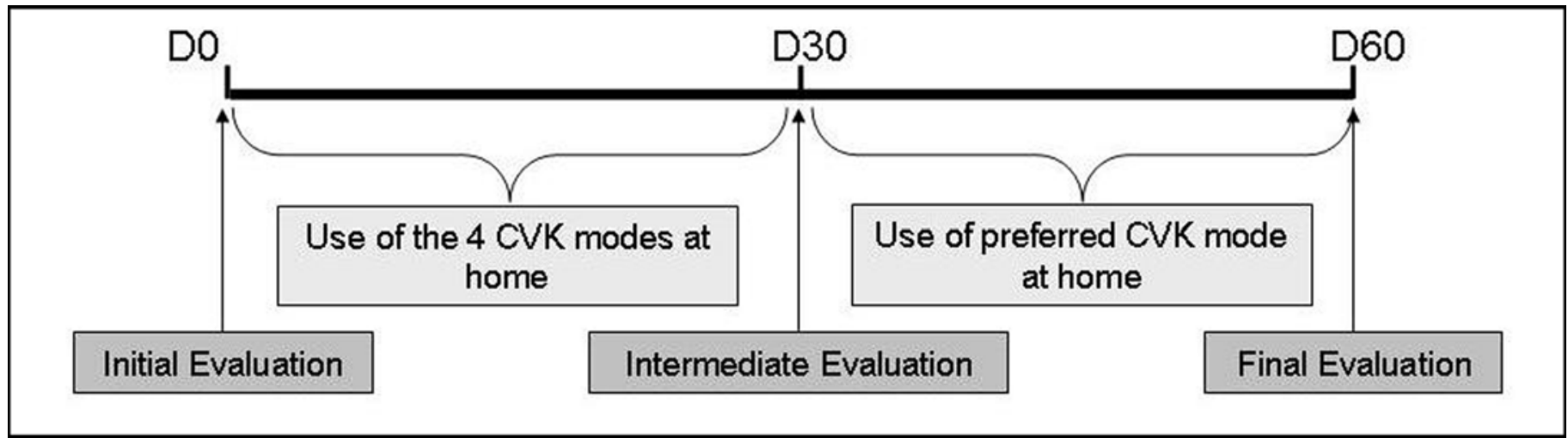

Figure 4.

Three evaluations and use at home. CVK = Custom Virtual Keyboard, D0 = baseline, D30 = end of first month, D60 = end of second month.

for 2 s. For participants who used a scanning system, the recording was stopped at the end of three runs without a selection.

\section{Data Analysis}

To compare the effect of the four modes on text input speed, we performed repeated-measures analyses of variance. Keyboard (static or dynamic), word prediction (yes or no), and evaluation (D0 and D30) were the factors included.

\section{RESULTS}

\section{Participants}

Ten participants ( 8 males and 2 females) with a mean \pm SD age of $37 \pm 10 \mathrm{yr}$ were included. Among them, four had locked-in syndrome, four had myopathies, and two had cervical spinal cord injuries (Table 1).

Of the 10 participants, 5 also used their home computer for work. Nine participants used a pointing device to access the computer and one participant used a scanning system (row-column pattern). Of the nine participants who used pointing devices, four used a headpointing device, four a specific type of pointer operated by the upper limb (e.g., joystick or trackball), and one an eye pointer. Mean \pm SD duration of use of the pointing device was $53 \pm 37 \mathrm{mo}$. The habitually used on-screen keyboard was a Windows on-screen keyboard for five participants, a keyboard available by free download for three participants, and a commercially available keyboard for two participants (all were static AZERTY on-
Table 1.

Participant characteristics.

\begin{tabular}{cclll}
\hline Participant & $\begin{array}{c}\text { Age } \\
\text { (yr) }\end{array}$ & Sex & \multicolumn{1}{c}{ Diagnosis } & Device \\
\hline P1 & 22 & Male & Myopathy & Pointing \\
P2 & 41 & Male & Locked-in syndrome & Pointing \\
P3 & 35 & Female & Locked-in syndrome & Pointing \\
P4 & 26 & Female & Myopathy & Pointing \\
P5 & 33 & Male & Myopathy & Pointing \\
P6 & 38 & Male & Locked-in syndrome & Pointing \\
P7 & 32 & Male & Myopathy & Pointing \\
P8 & 44 & Male & Tetraplegia & Pointing \\
P9 & 49 & Male & Tetraplegia & Pointing \\
S1 & 53 & Male & Locked-in syndrome & Scanning \\
\hline P= participant using pointing device, S = participant using linear scanning. \\
\hline
\end{tabular}

screen keyboards). Mean \pm SD duration of on-screen keyboard use was $67 \pm 67$ mo. All the participants had direct prior experience with word-prediction software but not with dynamic keyboards.

\section{Usage Time of Each Mode}

Table 2 shows the usage time of each mode by each participant. Mean \pm SD usage time over the 2 mo period was $100 \pm 105 \mathrm{~h}$. At the end of the first month (D30), three participants chose the static mode and six chose the static+word-prediction mode. The remaining participant was the participant who used linear scanning, and he chose the dynamic mode. No participants chose the dynamic+word-prediction mode.

Several participants did not use all four modes during the first month. One participant intensively used the static and static+word-prediction modes (Table 2). 
Table 2.

Each participant's usage time of Custom Virtual Keyboard (CVK) for four modes over 2 mo study period. Shown as hours (\% overall time) for first month and hours (chosen mode) for second month.

\begin{tabular}{|c|c|c|c|c|c|}
\hline \multirow[b]{2}{*}{ Participant } & \multicolumn{4}{|c|}{ First Month } & \multirow{2}{*}{ Second Month } \\
\hline & St & StW & $\bar{D}$ & DW & \\
\hline $\mathrm{P} 1$ & $0.3(5.3)$ & 3.8 (66.7) & $0.4(7.0)$ & $1.2(21.0)$ & $2.0(\mathrm{StW})$ \\
\hline P2 & $3.4(11.0)$ & $23.0(74.4)$ & 3.8 (12.3) & $0.7(2.3)$ & 21.5 (StW) \\
\hline P3 & $15.2(28.0)$ & $22.1(40.8)$ & $6.4(11.8)$ & $10.5(19.4)$ & $20.5(\mathrm{StW})$ \\
\hline $\mathrm{P} 4$ & 38.5 (78.7) & $10.0(20.5)$ & $0.1(0.2)$ & $0.3(0.6)$ & 29.5 (StW) \\
\hline P5 & $12.3(56.9)$ & $0.6(2.8)$ & $0.1(0.5)$ & $8.6(39.8)$ & $0.7(\mathrm{StW})$ \\
\hline P6 & $101.2(40.8)$ & $129.3(52.0)$ & $12.8(5.2)$ & $5.1(2.0)$ & $122.0(\mathrm{St})$ \\
\hline P7 & $41.2(74.2)$ & $0.1(0.2)$ & $1.9(3.4)$ & $12.3(22.2)$ & $44.4(\mathrm{St})$ \\
\hline P8 & $0.3(0.4)$ & $24.3(29.4)$ & $7.8(9.5)$ & $50.0(60.7)$ & $3.0(\mathrm{StW})$ \\
\hline P9 & 11.7 (19.4) & $48.6(80.5)$ & $0(0)$ & $0.1(0.1)$ & $20.1(\mathrm{St})$ \\
\hline S1 & $0.2(1.2)$ & $1.7(10.0)$ & $15.0(88.2)$ & $0.1(0.6)$ & 8.5 (D) \\
\hline
\end{tabular}

$\mathrm{D}$ = dynamic CVK mode, DW = dynamic+word-prediction CVK mode, $\mathrm{P}=$ participant using pointing device, $\mathrm{S}=$ participant using linear scanning, $\mathrm{St}=$ static $\mathrm{CVK}$ mode, StW = static+word-prediction CVK mode.

\section{Text Input Speed}

The optimal use of an unfamiliar on-screen keyboard may require a learning process. We performed longitudinal measurements to evaluate the effects of usage over time (Tables 3-4). There was no significant change in text input speed across evaluation sessions $(p=0.97$; Table 5). Neither were there any significant interactions between mode and evaluation session. Consequently, the results of the three evaluations were averaged.

\section{Effect of Mode on Text Input Speed}

Use of the dynamic keyboard decreased text input speed by a mean \pm SD of $37 \pm 27$ percent compared with use of the static keyboard (Figure 5). This reduction was statistically significant $(p=0.01)$ (Table 3$)$. Use of word prediction had no effect on text input speed $(p=0.82)$. There were no significant interactions between modes.

We identified no characteristics (i.e., age, sex, type of pointing device, diagnosis, usage time, or time since acqui-

Table 3.

Mean \pm standard deviation text input speed (characters/min) for evaluations at baseline (D0) and end of first month (D30) for all participants.

\begin{tabular}{lll}
\hline \multicolumn{1}{c}{ CVK Mode } & \multicolumn{1}{c}{ D0 } & \multicolumn{1}{c}{ D30 } \\
\hline Static & $23.4 \pm 12.9$ & $22.6 \pm 12.0$ \\
Static+Word Prediction & $23.0 \pm 12.3$ & $21.5 \pm 12.0$ \\
Dynamic & $11.9 \pm 4.9$ & $11.6 \pm 6.5$ \\
Dynamic+Word Prediction & $11.5 \pm 6.9$ & $12.9 \pm 7.6$ \\
\hline CVK = Custom Virtual Keyboard. & & \\
\hline \hline
\end{tabular}

Table 4.

Mean \pm standard deviation text input speed (characters/min) for evaluation at end of second month (D60).

\begin{tabular}{lll}
\hline \multicolumn{1}{c}{ CVK Mode } & \multicolumn{1}{c}{ D60 } & \multicolumn{1}{c}{ Participant } \\
\hline Static & $12.7 \pm 2.2$ & P6/P7/P9 \\
Static+Word Prediction & $24.3 \pm 11.3$ & P1/P2/P3/P4/P5/P8 \\
Dynamic & $5.5^{*}$ & S1 \\
Dynamic+Word Prediction & NA & None
\end{tabular}

${ }^{*}$ Only S1

CVK = Custom Virtual Keyboard, NA = not applicable, $\mathrm{P}$ = participant using pointing device, $\mathrm{S}=$ participant using linear scanning.

Table 5.

Analysis of variance for time input speed and Custom Virtual Keyboard modes.

\begin{tabular}{lc}
\hline \multicolumn{1}{c}{ Effect } & $\boldsymbol{p}$-Value \\
\hline Time (D0 vs D30) & 0.97 \\
Keyboard Type (Static vs Dynamic) & 0.01 \\
Word Prediction (With vs Without) & 0.82 \\
\hline D0 = baseline, D30 = end of first month. & \\
\hline \hline
\end{tabular}

sition of the pointing device) that appeared to be related to whether the dynamic keyboard or word-prediction tool increased or decreased text input speed.

\section{Participant Satisfaction}

Table 6 shows the level of satisfaction of each participant on the VAS. All nine participants who used pointing devices reported greater satisfaction with the static keyboard than with the dynamic keyboard. However, the 

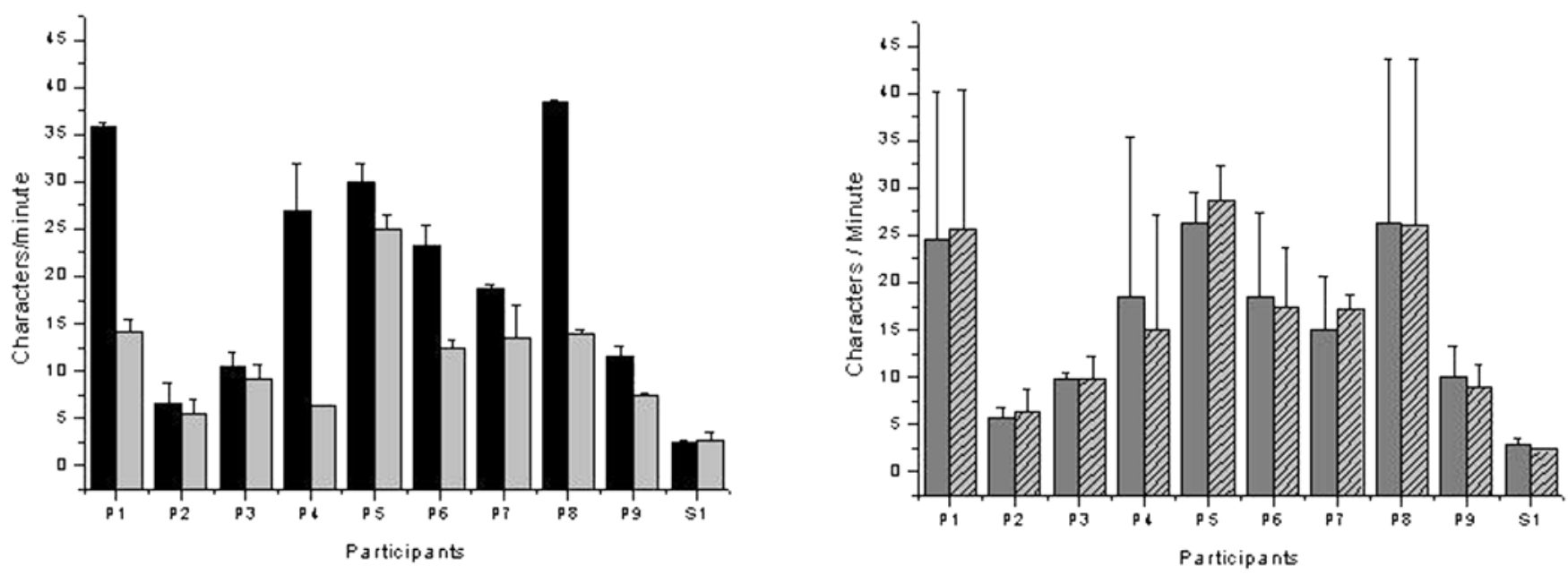

Figure 5.

Text input speed (mean \pm standard deviation characters/minute) of three evaluation sessions combined for each participant. Black bar = static on-screen keyboard, light gray bar = dynamic on-screen keyboard, dark gray bar = without word prediction, striped gray bar = with word prediction. $\mathrm{P}=$ participant using pointing device, $\mathrm{S}=$ participant using linear scanning.

participant who used linear scanning was more satisfied with the dynamic keyboard.

At the end of the study, 9 of the 10 participants reported that they preferred to keep their own on-screen keyboard. A single participant, who used a pointing device, wanted to keep the CVK (in the static+wordprediction mode) instead of the Windows XP keyboard he used previously.

Table 6.

Visual analog scale satisfaction scores (out of 10) at end of first month.

\begin{tabular}{ccccc}
\hline \multirow{2}{*}{ Participant } & \multicolumn{4}{c}{ CVK Mode } \\
\cline { 2 - 5 } & Static & $\begin{array}{c}\text { Static + Word } \\
\text { Prediction }\end{array}$ & Dynamic & $\begin{array}{c}\text { Dynamic + Word } \\
\text { Prediction }\end{array}$ \\
\hline P1 & 7 & $6^{*}$ & 2 & 3 \\
P2 & 5 & $6^{*}$ & 3 & 5 \\
P3 & 2 & $5^{*}$ & 2 & 0 \\
P4 & 5 & $4^{*}$ & 1 & 0 \\
P5 & 6 & $7^{*}$ & 5 & 4 \\
P6 & 7 & $7^{*}$ & 0 & 0 \\
P7 & $9^{*}$ & 8 & 4 & 4 \\
P8 & 7 & $6^{*}$ & 0 & 0 \\
P9 & $7^{*}$ & 6 & 3 & 3 \\
S1 & 5 & 6 & $7^{*}$ & 7 \\
\hline
\end{tabular}

*Mode chosen by each participant for second month of study.

$\mathrm{CVK}=$ Custom Virtual Keyboard, $\mathrm{P}=$ participant using pointing device, $\mathrm{S}=$ participant using linear scanning.

\section{DISCUSSION}

The primary aim of this study was to preliminarily evaluate how a dynamic on-screen keyboard and the addition of a word-prediction tool to a static and dynamic onscreen keyboard affected text input speed. We hypothesized that both word prediction and the dynamic keyboard would increase text input speed and, thus, the combination of both systems would further increase text input speed; however, the results showed that our hypotheses were false. The main findings were that use of the dynamic keyboard decreased text input speed compared with the static keyboard and the addition of word prediction neither increased nor decreased text input speed. Most participants preferred to return to their usual keyboards at the end of the study.

\section{Dynamic Versus Standard Keyboard}

Dynamic keyboards have existed for several years and are particularly used by people who use scanning systems [26,29] to increase text input speed and communication rate $[26,30]$, although they were also designed for people who use pointing devices [18-19,28]. In 2009-2010, our team developed a dynamic keyboard that was intended for use by users of both scanning systems and pointing devices [28]. 
The results of our study, although preliminary, suggest that dynamic keyboards may be ill-suited for participants who use pointing devices. Text input speed was decreased by the dynamic keyboard compared with the static keyboard, and only one participant (the participant who scanned) chose to continue using the dynamic keyboard during the second month of the trial, suggesting a lack of subjective benefit in most cases. However, our results contrast with those of Merlin and Reynal, who showed that their dynamic keyboard improved text input speed by 20 percent compared with a static QWERTY keyboard in six participants with disabilities who used pointing systems [19]. This difference may be explained by the fact that the type of prediction system used was different. In their system, the characters that had a low probably of being selected were replaced by those with a high probability, thus creating repetition of these characters across the keyboard and increasing the ease with which they could be selected [19]. In our keyboard, only the position of the character is altered according to its selection probability, requiring the subject to search for the desired character for each selection. Since the disposition of the characters cannot be learned, this may increase the cognitive load of the task [31].

Although there are very few studies on the effects of the design of dynamic keyboards on text input speed in subjects with disabilities, it is likely that the design is important. For example, the layout of static on-screen keyboards has been shown to affect text input speed in nondisabled subjects and subjects with disabilities [16,32-33]. Several studies have also shown that the keyboard layout affects text input speed in nondisabled subjects using scanning systems [31].

Despite the fact that the dynamic keyboard had no effect on his text input speed, the single participant who used linear scanning in our study chose to keep this device during the second study month. This suggests that there was a subjective advantage of this keyboard for this participant. The subjective benefits of dynamic keyboards have previously been described for participants with motor disability who use scanning systems [26]. This advantage of the dynamic keyboard when used with scanning systems requires confirmation in larger numbers of participants who use scanning systems, such as those with amyotrophic lateral sclerosis, locked-in syndrome, and advanced multiple sclerosis.

\section{Effect of Word Prediction}

The goal of word prediction is to increase text input speed by eliminating the need to select each letter in the word. Although it has been demonstrated that word prediction reduces the number of keystrokes, at least in nondisabled subjects (by 10.0\%-39.6\% when coupled with a dynamic keyboard and by $7.9 \%$ when coupled with a static keyboard) [31], the effects on text input speed are disparate. The results of our study showed that the addition of word prediction had no effect on text input speed. This result is similar to some results in the literature and contrasts with others. Closer examination of the literature suggests that the different effects of word prediction found may be related to the user population and/or the type of system it is coupled with. Studies in nondisabled subjects have found improvements of approximately 3 wpm when using word prediction with on-screen keyboards but not with standard keyboards [22,34]. Word prediction did not, however, appear to be effective in nondisabled subjects using a scanning system [24]. Koester and Levine found that word prediction slightly improved text input speed in nondisabled subjects using a mouth stick on a standard computer keyboard, while it significantly decreased text input speed (by a mean of $41 \%$ ) in subjects with high-level tetraplegia [23].

Other studies in participants with disabilities have also found negative results for the use of word prediction. A previous study by our group that evaluated the addition of word prediction in adults with cerebral palsy who used voice synthesizers found no significant improvement for 4 out of 10 participants [35]. In a series of studies involving individuals with spinal cord injury and persons with normal abilities, Koester and Levine found that the wordprediction system reduced the number of key selections necessary; however, each selection took significantly longer to make, leading them to suggest that the cognitive costs of using a word-prediction system overshadowed any potential benefit associated with the method, particularly for the group of subjects with disabilities [23,25].

The effect of word prediction might be influenced by several parameters. Different search strategies can influence input text speed, such as the number of letters the subject types before searching the list [36]. This was not evaluated in the present study because we gave no instructions to the participants with disabilities because we wished to assess spontaneous use. Further studies 
regarding this factor would provide useful information to therapists for training subjects with disabilities.

The number of predicted words provided is also likely to be an important factor because of the time required to scan the list. The Sibylle system displays five predicted words at a time. There is a trade-off between the time gained as a result of keystroke savings when using word prediction and the time lost in searching a list of predicted words [36]. A series of studies Koester and Levine suggests that each additional word in the list increases search time by $150 \mathrm{~ms}$ [23,25]. In a simulation study, Swiffin (1987) found that beyond six words, the list search time outweighed the keystroke savings [37]. However, at present, there are too few data relating to people with disabilities to determine the optimal number of words that should be displayed for such populations.

Another parameter that may influence the effect of word prediction is the position of the predicted-word list on the screen. We used two positions (a horizontal list above the static keyboard and a vertical list left of the dynamic keyboard), and although they are both frequently used, we do not know what their effect on text entry speed might be. Although there are some indications in the literature that the location of the prediction list might affect the accuracy of text entry and the ease of use of word prediction [38-39], the optimal position remains to be determined.

Interestingly, although word prediction did not improve text input speed, 7 of the 10 participants chose to continue using the word-prediction mode during the second study month, suggesting that they perceived a subjective benefit. They perhaps wanted to have the possibility to use it if they wished. Indeed, some expressed that "I can use it when I need to." Some participants also expressed difficulties in looking for words in the list while paying attention to the keyboard, the text to be copied, the text they were writing, etc., which reflects the notion of a high cognitive load.

\section{Participant Satisfaction}

At the end of the study, 9 of the 10 participants reported that they preferred to keep their own on-screen keyboard. We suggest that the reason for this is that the dynamic keyboard perturbed most of the users because they could not learn the position of the letters. With regard to the static keyboard evaluated, the participants already used static AZERTY keyboards and were more familiar with their own. There may also be an element of resistance to change to a new device, termed "path dependence.” For example, Dvorak et al. showed that the layout of the QWERTY keyboard was taken from the design of early typewriters and has not changed, despite arguments that other layouts may be more efficient or ergonomic [10]

\section{Limitations}

This study has several limitations. The time spent by each participant on each usage mode was not equal, which may have influenced the results. It is possible that with more practice on certain modes, there might have been more improvements. However, the fact that subjects chose not to use certain modes suggests that they did not find them helpful.

The word-prediction dictionary [40] and texts used [21] can also influence text input speed; however, we randomized the texts and Sibylle contains a large dictionary. We thus hope that any effect was limited.

Finally, we did not collect data regarding the use of word prediction in order to analyze the amount of use of the prediction list by each subject. How the prediction list is used can influence text input speed [41]. Equally, the predicted lists were positioned differently for the different keyboards (above and horizontal to the static keyboard and left and vertical to the dynamic keyboard). Although these positions are frequently used, we do not know if they have different effects on text input speed.

\section{CONCLUSIONS}

In this preliminary study, the dynamic keyboard and the addition of a word-prediction tool failed to improve text input speed compared with a static on-screen keyboard without word prediction in adults with functional tetraplegia who used pointing devices and scanning systems. These results highlight the importance of testing assistive systems in the participants' everyday setting to ensure that the product under development meets the needs of the future users. The results of this study also raise questions regarding many factors, such as the best ergonomic design of a dynamic keyboard and the optimal number and position of words that should be predicted. Future studies should aim to address these questions in larger numbers of participants who use scanning systems. 


\section{ACKNOWLEDGMENTS}

Author Contributions:

Conception and design of protocol: S. Pouplin, J. Bouteille. Analysis and interpretation of data: S. Pouplin, J. Robertson, D. Bensmail.

Drafting of manuscript: S. Pouplin, J. Robertson, J.-Y. Antoine, F. Lofaso, D. Bensmail.

Programmed Sibylle software: J.-Y. Antoine.

Programmed CVK software: A. Blanchet, J. L. Kahloun, P. Volle.

Financial Disclosures: The authors have declared that no competing interests exist.

Funding/Support: This material was based on work supported by the Association Française contre les Myopathies, Alcatel- Lucent, the Fondation Garches, and the Fondation Steria-Institut de France.

Institutional Review: This was a pilot study for which ethical approval was not necessary according to French law, because it was an evaluation of usual practice.

Participant Follow-Up: The authors do not plan to inform participants of the publication of this study because contact information is unavailable.

\section{REFERENCES}

1. Bigot R, Croutte P. La diffusion des technologies de l'information dans la société française. Rapport réalisé à la demande du Conseil Général des Technologies de l’Information (Ministère de l'Economie, des Finances et de l'Emploi) et de l'Autorité de Régulation des Communications Electroniques et des Postes [Internet]. Paris (France): Centre de Recherche pour l'Etude et l'Observation des Conditions de Vie (CREDOC); 2009. Available from http:// www.ladocumentationfrancaise.fr/rapports-publics/ 094000589/index.shtml. French.

2. Boonzaier D. The impact of assistive technologies on the lives of disabled people. In: Mokhtari M, editor. Independent living for persons with disabilities and elderly people. Assistive Technology Research Series. Vol. 12. Amsterdam (the Netherlands): IOS Press; 2003. p. 10-12.

3. Picard R, Souzy JP. Usage des TIC par les participants et les citoyens en situation de fragilité dans leurs lieux de vie, Rapport $\mathrm{n}^{\circ} \mathrm{I}-2.2-2007$. Paris (France): CGTI, Ministère de l'économie, des finances et de l'emploi; 2007. French.

4. Association Nationale pour le Logement des personnes Handicapées [Internet]; Des « TIC » pour tous; 2007. Brussels (Belgium): ANLH; 2007. Available from: http:// www.lesfamilles.be/ebooks/brochures tic fr/ TIC_Brochure_Fr.pdf. French.

5. DeVries RC, Deitz J, Anson D. A comparison of two computer access systems for functional text entry. Am J Occup Ther. 1998;52(8):656-65.

6. Chen YL, Chen WL, Kuo TS, Lai JS. A head movement image (HMI)-controlled computer mouse for people with disabilities. Disabil Rehabil. 2003;25(3):163-67.

[PMID:12648006]

http://dx.doi.org/10.1080/0963828021000024960

7. LoPresti EF, Brienza DM. Adaptive software for headoperated computer controls. IEEE Trans Neural Syst Rehabil Eng. 2004;12(1):102-11. [PMID:15068193] http://dx.doi.org/10.1109/TNSRE.2003.822762

8. Pouplin S, Biard N. Informatique et évolutions en termes de compensation de handicap d'origine motrice. In: Izard $\mathrm{MH}$, editor. Expérience en ergothérapie, vingt deuxième série, Rencontres en médecine physique et de réadaptation n¹5. Montpellier (France): Sauramps Médical; 2009. p. 286-94. French.

9. Le Pévédic B. Prédiction morphosyntaxique évolutive [doctoral thesis]. [Nantes]: Ecole doctorale Sciences pour l’ingénieur de Nantes; 1997.

10. Dvorak A, Merrick NL, Dealey WL, Ford GC. Typewriting behavior: Psychology applied to teaching and learning typewriting. New York (NY): American Book Company; 1936.

11. Raynal M, Vigouroux N. Genetic algorithm to generate optimized soft keyboard. Proceedings of Human Factors in Computing Systems Conference; 2005 Apr 2-7; Portland, OR. p. 1729-32.

12. Lesher GW, Moulton BJ. A method for optimizing singlefinger keyboards. Proceedings of Rehabilitation Engineering and Assistive Technology Society of North America Annual Conference; 2000 Jun 28-Jul 2; Orlando, FL. p. 91-93.

13. MacKenzie IS, Zhang SZ. The design and evaluation of a high performance soft keyboard. Proceedings of ACM Conference on Human Factors in Computing Systems; 1999 May 15-20; Pittsburgh, PA. p. 25-31.

14. Kushler C. AAC using a reduced keyboard. Proceedings of CSUN Conference on Technology for Persons with Disabilities; 1998 May; California State University, Northridge, $\mathrm{CA}$.

15. Lesher GW, Moulton BJ, Higginbotham DJ. Optimal character arrangements for ambiguous keyboards. IEEE Trans Rehabil Eng. 1998;6(4):415-23. [PMID:9865889] http://dx.doi.org/10.1109/86.736156

16. Vigouroux N, Vella F, Truillet P, Raynal M. Evaluation of AAC for text input by two groups of subjects: able-bodied subjects and disabled motor subjects. Proceedings of 8th European Research Consortium for Informatics and Mathematics Workshop "User Interfaces for All”; 2004 Jun 2829; Vienna, Austria.

17. Harbusch K, Kühn M. An evaluation study of two-button scanning with ambiguous keyboards. Proceedings of Actes 7th Conference for the Advancement of Assistive Technology in Europe; 2003; Dublin, Ireland.

18. Ward DJ, Blackwell AF, McKay DJ. A data-entry interface using continuous gestures and language model. Proceedings 
of 13th Annual ACM Symposium on User Interface Software and Technology; 2000 Nov 6-8; San Diego, CA.

19. Merlin B, Raynal M. Evaluation of Spread Key System with motor impaired users. Proceedings of International Conference on Computers Helping People with Special Needs; 2010 Jul 14-16; Vienna, Austria; Berlin (Germany): Springer; 2010.

20. Schadle I, Antoine J-Y, Le Pévedic B, Poirier F. Sibylettre: Prédiction de lettre pour la communication assistée. Revue d'Interaction Homme-Machine. 2002;3(2):115-33. French.

21. Higginbotham DJ. Evaluation of keystroke savings across five assistive communication technologies. Augment Altern Commun. 1992;8(4):258-72. http://dx.doi.org/10.1080/07434619212331276303

22. Anson D. The effect of word prediction on typing speed. Am J Occup Ther. 1993;47(11):1039-42. [PMID:8279499] http://dx.doi.org/10.5014/ajot.47.11.1039

23. Koester HH, Levine SP. Effect of a word prediction feature on user performance. Augment Altern Commun. 1996;12:15568. http://dx.doi.org/10.1080/07434619612331277608

24. Koester HH, Levine SP. Learning and performance of ablebodied individuals using scanning systems with and without word prediction. Assist Technol. 1994;6(1):42-53. [PMID:10147209] http://dx.doi.org/10.1080/10400435.1994.10132226

25. Koester HH, Lévine SP. Learning and performance in scanning systems with and without word prediction-report on a pilot study. Proceedings of Rehabilitation Engineering and Assistive Technology Society of North America International Conference; 1992; Washington, DC. p. 299-301.

26. Heckathorne C, Voda J, Leibowitz L. Design rationale and evaluation of the portable anticipatory communication aid-PACA. Augment Altern Commun. 1987;3:170-80. http://dx.doi.org/10.1080/07434618712331274489

27. Wandmacher T, Antoine JY, Schadle I, Krueger-Thielmann K. Sibylle AAC system: exploiting syntax and semantics for word prediction. Proceedings of 12th Biennial Conference of International Society for Augmentative and Alternative Communication; 2006 Jul; Düsseldorf, Germany.

28. Wandmacher T, Antoine JY, Departe JP, Poirier F. SIBYLLE, an assistive communication system adapting to the context and its user. ACM Trans Access Comput. 2008; 1(1):1-30. http://dx.doi.org/10.1145/1361203.1361209

29. Gibler C, Childress D. Language anticipation with a computer-based scanning communication aid. Proceedings of Institute of Electrical and Electronics Engineers Computer Society Workshop on Computing to Aid the Handicapped; 1982; Charlottesville, VA. p. 11-16.

30. Baletsa G, Foulds R, Crochetiere W. Design parameters of an intelligent communication device. Proceedings of 29th Annual Conference on Engineering in Medicine and Biology; 1976; Chevy Chase, MD. p. 371.
31. Lesher GW, Bryan JM. Techniques for augmenting scanning communication. Augment Altern Commun. 1998; 14(2):81-101. http://dx.doi.org/10.1080/07434619812331278236

32. Raynal M., Vigouroux N. KeyGlasses: Semi-transparent keys to optimize text input on virtual keyboard. Proceedings of Advancement of Assistive Technology in Europe 2005; 2005 Sep 6-9; Lille, France. p. 713-17.

33. Vigouroux N, Vella F, Raynal M, Boissière P. Solutions et défis pour une meilleure accessibilité et utilisabilité des communicateurs: "Optimisation de la saisie de texte ", Actes des Entretiens de la Fondation Garches: « Handicap et Environnement: de l'adaptation du logement à l'accessibilité de la cité ». Paris (France): éditions Frison-Roche; 2005. p. 213-26. French.

34. Anson D, Moist P, Przywara M, Wells H, Saylor H, Maxime $\mathrm{H}$. The effects of word completion and word prediction on typing rates using on-screen keyboards. Assist Technol. 2006;18(2):146-54. [PMID:17236473]

http://dx.doi.org/10.1080/10400435.2006.10131913

35. Laffont I, Dumas C, Pozzi D, Ruquet M, Tissier AC, Lofaso F, Dizien O. Home trials of a speech synthesizer in severe dysarthria: Patterns of use, satisfaction and utility of word prediction. J Rehabil Med. 2007;39(5):399-404.

[PMID:17549332]

http://dx.doi.org/10.2340/16501977-0056

36. Koester HH, Levine SP. Model simulations of user performance with word prediction. Augment Altern Commun. 1998;14(1):25-36.

http://dx.doi.org/10.1080/07434619812331278176

37. Swiffin AL, Arnott JL, Pickering JA, Newell AF. Adaptive and predictive techniques in a communication prosthesis. Augment Altern Commun. 1987;3:181-91.

http://dx.doi.org/10.1080/07434618712331274499

38. Tam C, Reid D, Naumann S, O’Keefe B. Effects of word prediction and location of word prediction list on text entry with children with spina bifida and hydrocephalus. Augment Altern Commun. 2001;17(3):147-62.

39. Tam C, Wells D. Evaluating the benefits of displaying word prediction lists on a personal digital assistant at the keyboard level. Assist Technol. 2009;21(3):105-14.

[PMID:19908678] http://dx.doi.org/10.1080/10400430903175473

40. Higginbotham DJ, Bisantz AM, Sunm M, Adams K, Yik F. The effect of context priming and task type on augmentative communication performance. Augment Altern Commun. 2009;25(1):19-31. [PMID:18608144] http://dx.doi.org/10.1080/07434610802131869

41. Trnka K, McCaw J, Yarrington D, McCoy KF, Pennington C. User interaction with word prediction: the effects of prediction quality. ACM Trans Access Comput. 2009;1(3):1-34. http://dx.doi.org/10.1145/1497302.1497307 
Submitted for publication June 11, 2012. Accepted in revised form November 15, 2013.

This article and any supplementary material should be cited as follows:

Pouplin S, Robertson J, Antoine J, Blanchet A, Kahloun JL, Volle P, Bouteille J, Lofaso F, Bensmail D. Effect of dynamic keyboard and word-prediction systems on text input speed in persons with functional tetraplegia. J Rehabil Res Dev. 2014;51(3):467-80.

http://dx.doi.org/10.1682/JRRD.2012.05.0094

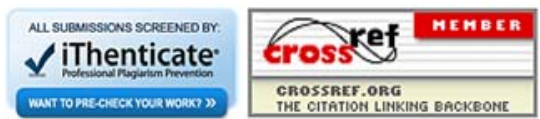


